\title{
Parity in Bloch's conductor formula in even dimension
}

\author{
par TAKESHI SAITO
}

\begin{abstract}
RÉSUMÉ. Pour une variété sur un corps local, Bloch a proposé une formule conjecturale pour la somme alternée du conducteur d'Artin de la cohomologie $\ell$-adique. On démontre que la formule modulo 2 est vraie dans le cas où la dimension de la variété est paire.
\end{abstract}

ABSTRACT. For a variety over a local field, Bloch proposed a conjectural formula for the alternating sum of Artin conductor of $\ell$ adic cohomology. We prove that the formula is valid modulo 2 if the variety has even dimension.

\section{Introduction}

Let $K$ be a complete discrete valuation field with perfect residue field $F$ of arbitrary characteristic and $X$ be a proper flat regular scheme over the integer ring $O_{K}$ with smooth generic fiber $X_{K}$. The Artin conductor $\operatorname{Art}\left(X / O_{K}\right)$ is defined by

$$
\operatorname{Art}\left(X / O_{K}\right)=\chi\left(X_{\bar{K}}\right)-\chi\left(X_{\bar{F}}\right)+\operatorname{Sw} H^{*}\left(X_{\bar{K}}, \mathbb{Q}_{\ell}\right) .
$$

Each term in the right hand side is defined as follows. We take a prime number $\ell$ different from the characteristic of $F$. Let $K^{\mathrm{nr}}$ be the maximal unramified extension of $K$ in an separable closure $\bar{K}$ and let $\bar{F}$ be the residue field of $K^{\mathrm{nr}}$. The first two terms

$$
\chi\left(X_{\bar{K}}\right)=\sum_{q}(-1)^{q} \operatorname{dim} H^{q}\left(X_{\bar{K}}, \mathbb{Q}_{\ell}\right), \chi\left(X_{\bar{F}}\right)=\sum_{q}(-1)^{q} \operatorname{dim} H^{q}\left(X_{\bar{F}}, \mathbb{Q}_{\ell}\right)
$$

are the $\ell$-adic Euler number of the geometric generic fiber $X_{\bar{K}}$ and of the geometric special fiber $X_{\bar{F}}$ respectively. The last term

$$
\text { Sw } H^{*}\left(X_{\bar{K}}, \mathbb{Q}_{\ell}\right)=\sum_{q}(-1)^{q} \mathrm{Sw} H^{q}\left(X_{\bar{K}}, \mathbb{Q}_{\ell}\right)
$$

denotes the alternating sum of the Swan conductor of the $\ell$-adic representations $H^{q}\left(X_{\bar{K}}, \mathbb{Q}_{\ell}\right)$ of the inertia subgroup $I=\operatorname{Gal}\left(\bar{K} / K^{\mathrm{nr}}\right)=\operatorname{Ker}\left(G_{K}=\right.$ $\left.\operatorname{Gal}(\bar{K} / K) \rightarrow G_{F}=\operatorname{Gal}(\bar{F} / F)\right)$. Each term in the right hand side is known to be independent of a choice of prime number $\ell[9]$. 
Bloch defines the self-intersection cycle $\left(\Delta_{X}, \Delta_{X}\right)_{s}$ as a 0 -cycle class supported on the closed fiber $X_{F}$. It is defined as a localized chern class

$$
\left(\Delta_{X}, \Delta_{X}\right)_{s}=(-1)^{n+1} c_{n+1} X_{F}^{X}\left(\Omega_{X / O_{K}}^{1}\right) \in C H_{0}\left(X_{F}\right) .
$$

Here $n=\operatorname{dim} X_{K}$ is the dimension.

Conjecture 0.1. [1] Let $X$ be a proper flat regular scheme over a complete discrete valuation ring $O_{K}$ with perfect residue field $F$ such that the generic fiber $X_{K}$ is smooth. Then we have

$$
\operatorname{deg}\left(\Delta_{X}, \Delta_{X}\right)_{s}=-\operatorname{Art}\left(X / O_{K}\right) .
$$

Conjecture is proved by Bloch [1] for curves, $\operatorname{dim} X_{K}=1$.

We prove the following assertion on the parity for varieties of even dimension.

Theorem 0.2. Let $X$ be a projective flat regular scheme over a complete discrete valuation ring $O_{K}$ with perfect residue field $F$ such that the generic fiber $X_{K}$ is smooth. We assume that the dimension $n=\operatorname{dim} X_{K}$ is even. We assume further that the reduced closed fiber $X_{F, \text { red }}$ is a divisor of $X$ with normal crossing and that the characteristic of $K$ is not 2. Then, we have a congruence

$$
\operatorname{deg}\left(\Delta_{X}, \Delta_{X}\right)_{s} \equiv \operatorname{Art}\left(X / O_{K}\right) \quad \bmod 2 .
$$

The outline of proof is the following. We consider the highest exterior power $\operatorname{det} H^{n}\left(X_{\bar{K}}, \mathbb{Q}_{\ell}\right)$ of the cohomology of middle dimension. It defines a character of the inertia group $I$ of order at most 2 and its Artin conductor $\operatorname{Art}\left(\operatorname{det} H^{n}\left(X_{\bar{K}}, \mathbb{Q}_{\ell}\right)\right)$ is defined. We also consider the de Rham cohomology $H_{d R}^{n}\left(X_{K} / K\right)$ of middle dimension. The cup-product defines a symmetric non-degenerate bilinear form on it. Hence its discriminant $\operatorname{disc} H_{d R}^{n}\left(X_{K} / K\right)$ is defined as an element of $K^{\times} / K^{\times 2}$. Its valuation ord $\left(\operatorname{disc} H_{d R}^{n}\left(X_{K} / K\right)\right)$ is well-defined as an integer modulo 2 . We prove Theorem by showing congruences

$$
\begin{aligned}
\operatorname{deg}\left(\Delta_{X}, \Delta_{X}\right) & \stackrel{(0.1)}{\equiv} \operatorname{ord}\left(\operatorname{disc} H_{d R}^{n}\left(X_{K} / K\right)\right) \\
& \stackrel{(0.2)}{\equiv} \operatorname{Art}\left(\operatorname{det} H^{n}\left(X_{\bar{K}}, \mathbb{Q}_{\ell}\right)\right) \\
& \stackrel{(0.3)}{\equiv} \operatorname{Art}\left(X / O_{K}\right) \quad \bmod 2 .
\end{aligned}
$$

In section 1 , we deduce the congruence (0.3), Proposition 1.1, from the following two facts. One is the computation of vanishing cycles given in [3], which is a consequence of absolute purity recently proved by O.Gabber [5]. The other is a theorem of Serre [12] on the parity of Artin conductor of an orthogonal representation. 
In section 2, we derive the congruence (0.1), Proposition 2.1, from a Riemann-Roch formula, Corollary 4.9, for the derived exterior power complex $L \Lambda^{q} \Omega_{X / O_{K}}^{1}$ of the coherent sheaf $\Omega_{X / O_{K}}^{1}$. The formula is an analogue of a formula of Bloch [2] in higher dimension and proved similarly as in [10]. Its proof is given later in Section 4 after recalling the definition and some properties of the derived exterior power complexes.

The congruence $(0.2)$ is an immediate consequence of the relation, Theorem 2 [11] recalled in section 3 , between the determinant of $\ell$-adic etale cohomology and the discriminant of the de Rham cohomology.

In the final section 4 , we study the exterior derived power complex and slightly generalize the results in [10]. Using it, we prove the Riemann-Roch formula, Corollary 4.9, and complete the proof of the congruence (0.1) and of Theorem 0.2 .

There is an error in the definition of the exterior derived power complex given in [10] but it has no influence on the proof of the results proved there. We give a correct definition in Definition 4.1 in Section 4 and give in Proof of Proposition 4.8 an argument why it has no influence. The author apologizes for the mistake.

Shortly after the conference at Luminy, in a joint work with K.Kato, the author found a proof of the conductor formula, Conjecture 0.1, under the assumption that the reduced closed fiber is a divisor with simple normal crossings. The proof will be published in [8].

Acknowledgement The author would like to thank Prof. S. Bloch for asking the proof of the congruence (0.1) above. It inspired the auther greatly to find the proof of Theorem. He also thanks to Prof. H. Esnault for stimulating discussion and for encouragement. The author is grateful to Profs. L. Illusie and A. Abbes and to the anonymous referee for careful reading of an earlier version of the article.

\section{Parity of conductor}

In this section, $O_{K}$ denotes a complete discrete valuation ring with perfect residue field $F$. We prove the congruence (0.3).

Proposition 1.1. Let $X$ be a proper flat regular scheme over $O_{K}$ such that the generic fiber $X_{K}$ is smooth. We assume that the dimension $n=\operatorname{dim} X_{K}$ is even. If we further assume that the reduced closed fiber $X_{F, \text { red }}$ is a divisor of $X$ with normal crossing, we have a congruence

$$
\operatorname{Art}\left(X / O_{K}\right) \equiv \operatorname{Art}\left(\operatorname{det} H^{n}\left(X_{\bar{K}}, \mathbb{Q}_{\ell}\right)\right) \quad \bmod 2 .
$$

We introduce some notation to prove Proposition 1 . Let $V$ be a quasiunipotent $\ell$-adic representation of $I$. The $\operatorname{Artin}$ conductor $\operatorname{Art}(V)$ is defined by

$$
\operatorname{Art}(V)=\operatorname{dim} V-\operatorname{dim} V^{I}+\operatorname{Sw}(V)
$$


where $V^{I}$ denotes the $I$-fixed part and $\operatorname{Sw}(V)$ denotes the Swan conductor of $V$. We define the semi-simplified version $\operatorname{Art}^{\text {ss }}(V)$ as follows. Take an $I$ invariant filtration $F$ on $V$ such that the action of $I$ on $\mathrm{Gr}_{\bullet}^{F} V=\bigoplus_{q} \operatorname{Gr}_{q}^{F} V$ factors a finite quotient of $I$. For example we may take the monodromy filtration. Then the dimension $\operatorname{dim}\left(\mathrm{Gr}_{\bullet}^{F} V\right)^{I}$ of the $I$-fixed part $\left(\mathrm{Gr}_{\bullet}^{F} V\right)^{I}$ is independent of the choice of $F$. We denote it by $\operatorname{dim}_{s} V$. For a quasiunipotent $\ell$-adic representation $V$ of $I$, we define the semi-simplified Artin conductor $\operatorname{Art}^{\mathrm{sS}}(V)$ by

$$
\operatorname{Art}^{\mathrm{ss}}(V)=\operatorname{dim} V-\operatorname{dim}_{s} V+\operatorname{Sw} V .
$$

If we take a filtration $F$ on $V$ as above, we have $\operatorname{Art}^{\mathrm{ss}}(V)=\operatorname{Art}\left(\operatorname{Gr}_{\bullet}^{F} V\right)$. For a proper smooth scheme $X_{K}$ over $K$, we put

$$
\chi_{s}\left(X_{\bar{K}}\right)=\sum_{q}(-1)^{q} \operatorname{dim}_{s} H^{q}\left(X_{\bar{K}}, \mathbb{Q}_{\ell}\right) .
$$

We reduce Proposition 1.1 to the following Lemmas.

Lemma 1.2. For $X$ as in Proposition 1, we have

$$
\chi\left(X_{\bar{F}}\right) \equiv \chi_{s}\left(X_{\bar{K}}\right) \quad \bmod 2 .
$$

Lemma 1.3. For an orthogonal representation $V$ of $I$, the semi-simplified Artin conductor $\operatorname{Art}^{\text {ss }}(V)$ is congruent to the Artin conductor $\operatorname{Art}(\operatorname{det} V)$ of the determinant representation $\operatorname{det} V$ :

$$
\operatorname{Art}^{s s}(V) \equiv \operatorname{Art}(\operatorname{det} V) \quad \bmod 2 .
$$

We deduce Proposition 1.1 from Lemmas 1.2 and 1.3. It is enough to prove congruences

$$
\begin{aligned}
\operatorname{Art}\left(X / O_{K}\right) & =\chi\left(X_{\bar{K}}\right)-\chi\left(X_{\bar{F}}\right)+\operatorname{Sw} H^{*}\left(X_{\bar{K}}, \mathbb{Q}_{\ell}\right) \\
& \stackrel{(1.1)}{\equiv} \chi\left(X_{\bar{K}}\right)-\chi_{s}\left(X_{\bar{K}}\right)+\operatorname{Sw} H^{*}\left(X_{\bar{K}}, \mathbb{Q}_{\ell}\right) \\
& \stackrel{(1.2)}{\equiv} \operatorname{Art}^{\mathrm{ss}}\left(H^{n}\left(X_{\bar{K}}, \mathbb{Q}_{\ell}\right)\right) \\
& \stackrel{(1.3)}{\equiv} \operatorname{Art}\left(\operatorname{det} H^{n}\left(X_{\bar{K}}, \mathbb{Q}_{\ell}\right)\right) \quad \bmod 2 .
\end{aligned}
$$

The congruences (1.1) and (1.3) are Lemmas 1.2 and 1.3 respectively. The congruence (1.2) follows from the equality

$$
\begin{aligned}
\chi\left(X_{\bar{K}}\right)-\chi_{s}\left(X_{\bar{K}}\right)+\operatorname{Sw} H^{*}\left(X_{\bar{K}}, \mathbb{Q}_{\ell}\right)=\sum_{q=0}^{2 n}(-1)^{q} \operatorname{Art}^{\mathrm{ss}} H^{q}\left(X_{\bar{K}}, \mathbb{Q}_{\ell}\right) \\
=(-1)^{n} \operatorname{Art}^{\mathrm{ss}} H^{n}\left(X_{\bar{K}}, \mathbb{Q}_{\ell}\right)+2 \sum_{q=0}^{n-1}(-1)^{q} \operatorname{Art}^{\mathrm{ss}} H^{q}\left(X_{\bar{K}}, \mathbb{Q}_{\ell}\right) .
\end{aligned}
$$


The last equality follows from Poincaré duality and the equality $\mathrm{Art}^{\mathrm{ss}} V=$ $\mathrm{Art}^{\mathrm{ss}} V^{*}$ for the dual representation $V^{*}$.

Proof of Lemma 1.2. We prove a more precise statement Lemma 1.4 below. We may and do assume $F=\bar{F}$ is an algebraically closed field.

We change the notation in the rest of this section as follows. We assume $F=\bar{F}$ is algebraically closed and we drop the assumption that the dimension $n=\operatorname{dim} X_{K}$ is even. Let $Y=X_{F, \text { red }}$ denote the reduced closed fiber. We define proper smooth schemes $Y^{(k)}$ over $F$ for $0 \leq k \leq n=\operatorname{dim} X_{K}$ of dimension $n-k$. When $Y$ has simple normal crossing, it is the disjoint union of $(k+1) \times(k+1)$-intersections of irreducible components of $Y$. We define $Y^{(0)}$ to be the normalization of $Y$. For $k \geq 1$, we define $Y^{(k)}$ as follows. We put

$$
\left(\prod_{Y}^{k+1} Y^{(0)}\right)^{o}=\prod_{Y}^{k+1} Y^{(0)}-\bigcup_{0 \leq i<j \leq k} \Delta_{i j}
$$

Here $\prod_{Y}^{k+1} Y^{(0)}=Y^{(0)} \times_{Y} Y^{(0)} \times_{Y} \cdots \times_{Y} Y^{(0)}$ denotes the $k+1$-fold self fiber product. For $0 \leq i<j \leq k$, the closed subscheme $\Delta_{i j}=\left(y_{i}=y_{j}\right)$ is the image of a closed immersion

$$
\prod_{Y}^{k} Y^{(0)} \rightarrow \prod_{Y}^{k+1} Y^{(0)}:\left(y_{0}, \ldots, y_{k}\right) \mapsto\left(y_{0}, \ldots, y_{j-1}, y_{i}, y_{j}, \ldots, y_{k}\right) .
$$

The natural action of the symmetric group $\mathcal{S}_{k+1}$ on $\left(\prod_{Y}^{k+1} Y^{(0)}\right)^{o}$ is free and we define $Y^{(k)}$ to be the quotient $\left(\prod_{Y}^{k+1} Y^{(0)}\right)^{o} / \mathcal{S}_{k+1}$. From the assumption that $Y$ has normal crossing, it follows that $Y^{(k)}$ is smooth of dimension $n-k$ and the canonical map $Y^{(k)} \rightarrow Y$ is finite.

To show Lemma 1.2, we prove more precisely

Lemma 1.4. Let $X$ be a proper regular flat scheme over a discrete valuation ring $O_{K}$ with algebraically closed residue field $F$ such that the generic fiber $X_{K}$ is smooth of arbitrary dimension $n=\operatorname{dim} X_{K}$. We assume that the reduced closed fiber $Y=X_{F, \text { red }}$ is a divisor with normal crossings and define the schemes $Y^{(k)}$ as above. Then, we have equalities

$$
\begin{aligned}
\chi(Y) & =\sum_{k=0}^{n}(-1)^{k} \chi\left(Y^{(k)}\right), \\
\chi_{s}\left(X_{\bar{K}}\right) & =\sum_{k=0}^{n}(-1)^{k}(k+1) \chi\left(Y^{(k)}\right) .
\end{aligned}
$$

Lemma 1.4 implies Lemma 1.2. In fact, if $n=\operatorname{dim} X_{K}$ is even and $k$ is odd, the scheme $Y^{(k)}$ is proper smooth of odd dimension $n-k$. Hence by Poincaré duality, the Euler number $\chi\left(Y^{(k)}\right)$ is even. 
Proof of Lemma 1.4. 1. Let $L^{(k)}$ be the locally constant sheaf of rank 1 on $Y^{(k)}$ corresponding to the character

$$
\operatorname{sgn}: \mathcal{S}_{(k+1)}=\operatorname{Gal}\left(\left(\prod_{Y}^{k+1} Y^{(0)}\right)^{o} / Y^{(k)}\right) \rightarrow\{ \pm 1\} \subset \mathbb{Q}_{\ell}^{\times}
$$

and let $\pi^{(k)}: Y^{(k)} \rightarrow Y$ denote the canonical map. Then we have an exact sequence

$$
\begin{aligned}
0 \longrightarrow \mathbb{Q}_{\ell} \longrightarrow \pi_{*}^{(0)} L^{(0)} \longrightarrow & \pi_{*}^{(1)} L^{(1)} \longrightarrow \\
\ldots \quad \longrightarrow & \longrightarrow \pi_{*}^{(n)} L^{(n)} \longrightarrow 0
\end{aligned}
$$

of sheaves on $Y$. By the Lefschetz trace formula, we easily obtain the equality $\chi\left(Y^{(k)}, L^{(k)}\right)=\chi\left(Y^{(k)}\right)$. Thus the assertion 1 is proved.

2. Since the absolute purity is proved by Gabber [5], the tame part $R_{t}^{q}=R^{q} \psi^{P} \mathbb{Q}_{\ell}$ of the vanishing cycle is computed as in [3]. The result loc.cit. is summarized as follows. Define a map $\mathbb{Q}_{\ell}(-1) \rightarrow \pi_{*}^{(0)} \mathbb{Q}_{\ell}(-1)$ to be the multiplication by the prime-to- $p$ part of the multiplicity in the closed fiber $X_{F}$ to the component corresponding to each irreducible component of $Y^{(0)}$ and put $R=\operatorname{Coker}\left(\mathbb{Q}_{\ell}(-1) \rightarrow \pi_{*}^{(0)} \mathbb{Q}_{\ell}(-1)\right)$. Then the cup-product induces an isomorphism $R_{t}^{0} \otimes \Lambda^{q} R \rightarrow R_{t}^{q}$ for all integer $q \geq 0$. Further, the action of the tame quotient $I / P$ on $R_{t}^{0}$ factors a finite quotient and the fixed part $R_{s}^{0}=\left(R_{t}^{0}\right)^{I / P}$ is equal to the constant sheaf $\mathbb{Q}_{\ell}$. Consequently, the fixed part $R_{s}^{q}=\left(R_{t}^{q}\right)^{I / P}$ is identified with $\Lambda^{q} R$.

Since the action of $I / P$ on $R^{q} \psi^{P} \mathbb{Q}_{\ell}$ factors a finite quotient, by the spectral sequence $H^{p}\left(Y, R^{q} \psi^{P} \mathbb{Q}_{\ell}\right) \Rightarrow H^{p+q}\left(X_{\bar{K}}, \mathbb{Q}_{\ell}\right)^{P}$, we have

$$
\chi_{s}\left(X_{\bar{K}}\right)=\sum_{q}(-1)^{q} \chi\left(Y, R_{s}^{q}\right) \text {. }
$$

By the description of $R_{s}^{q}$ above we get an exact sequence

$$
\begin{aligned}
0 \longrightarrow \mathbb{Q}_{\ell} \longrightarrow \pi_{*}^{(0)} L^{(0)} \longrightarrow & \longrightarrow \pi_{*}^{(1)} L^{(1)} \longrightarrow \\
\cdots & \longrightarrow \pi_{*}^{(q-1)} L^{(q-1)} \longrightarrow R_{s}^{q}(q) \longrightarrow 0
\end{aligned}
$$

of sheaves on $Y$. Hence together with the equality (1), we have

$$
\chi(Y)=\sum_{k=0}^{q-1}(-1)^{k} \chi\left(Y^{(k)}\right)+(-1)^{q} \chi\left(Y, R_{s}^{q}\right)=\sum_{k=0}^{n}(-1)^{k} \chi\left(Y^{(k)}\right)
$$

and hence $(-1)^{q} \chi\left(Y, R_{s}^{q}\right)=\sum_{k=q}^{n}(-1)^{k} \chi\left(Y^{(k)}\right)$. Substituting this in the equality (3) above, we get the equality (2).

Proof of Lemma 1.3. We consider the monodromy filtration $W$ on $V$. Then each graded piece $\mathrm{Gr}_{r}^{W} V$ is isomorphic to $\mathrm{Gr}_{-r}^{W} V$ and hence to the 
dual $\left(\operatorname{Gr}_{r}^{W} V\right)^{*}$. Hence we have

$$
\begin{gathered}
\operatorname{Art}^{\mathrm{ss}}(V)=\operatorname{Art}\left(\mathrm{Gr}_{0}^{W} V\right)+2 \sum_{r>0} \operatorname{Art}\left(\mathrm{Gr}_{r}^{W} V\right) \equiv \operatorname{Art}\left(\mathrm{Gr}_{0}^{W} V\right) \bmod 2, \\
\operatorname{det} V \simeq \operatorname{det}\left(\mathrm{Gr}_{0}^{W} V\right) \otimes \bigotimes_{r>0}\left(\operatorname{det}\left(\mathrm{Gr}_{r}^{W} V\right) \otimes \operatorname{det}\left(\left(\mathrm{Gr}_{r}^{W} V\right)^{*}\right)\right) \simeq \operatorname{det}\left(\mathrm{Gr}_{0}^{W} V\right) .
\end{gathered}
$$

Thus it is enough to apply a theorem of Serre [12] to an orthogonal representation $\operatorname{Gr}_{0}^{W} V$ on which the action of the inertia group $I$ factors a finite quotient.

The following Lemma will not be used in the sequel.

Lemma 1.5. For an orthogonal representation $V$ of $I$, the semi-simplified Artin conductor $\operatorname{Art}^{s s}(V)$ is congruent to the Artin conductor $\operatorname{Art}(V)$ modulo 2:

$$
\operatorname{Art}^{s s}(V) \equiv \operatorname{Art}(V) \quad \bmod 2 .
$$

Proof. It is sufficient to show the congruence $\operatorname{dim}_{s} V \equiv \operatorname{dim} V^{I} \bmod 2$. We consider the monodromy filtration $W$ on $V$. It is enough to show the congruence $\operatorname{dim}\left(\mathrm{Gr}_{\bullet}^{W} V\right)^{I} \equiv \operatorname{dim} V^{I} \bmod 2$. By the definition of the monodromy filtration, we have $\operatorname{dim}\left(\mathrm{Gr}_{\bullet}^{W} V\right)^{I} \equiv \operatorname{dim}\left(\mathrm{Gr}_{0}^{W} V\right)^{I} \bmod 2$. Since we have $V^{I}=(\operatorname{Ker}(N: V \rightarrow V))^{I}$, it is enough to show the congruence $\operatorname{dim}\left(\mathrm{Gr}_{0}^{W} V\right)^{I} \equiv \operatorname{dim}(\operatorname{Ker}(N: V \rightarrow V))^{I} \bmod 2$.

For $r \geq 0$, let $P_{r}=\operatorname{Ker}\left(N^{r+1}: G r_{r}^{W} V \rightarrow G r_{-r-2}^{W} V\right)$ be the primitive part. Then we have isomorphisms

$$
\begin{aligned}
\oplus_{r} N^{r / 2}: \bigoplus_{r \geq 0, \text { even }} P_{r} & \longrightarrow \operatorname{Gr}_{0}^{W} V, \\
\oplus_{r} N^{r}: \bigoplus_{r \geq 0} P_{r} & \longrightarrow \operatorname{Ker}(N: V \rightarrow V) .
\end{aligned}
$$

Therefore, it is sufficient to show that the $\operatorname{dimension} \operatorname{dim} P_{r}^{I}$ of the $I$ fixed part $P_{r}^{I}$ is even for an odd integer $r>0$. It follows from that the primitive part $P_{r}$ for odd $r$ has an $I$-invariant non-degenerate alternating form $\left(x, N^{r} y\right)$.

\section{Parity of discriminant}

We prove the congruence $(0.1)$.

Proposition 2.1. Let $X$ be a projective flat regular scheme over a complete discrete valuation ring $O_{K}$ with perfect residue field such that the generic fiber $X_{K}$ is smooth of even dimension $n=\operatorname{dim} X_{K}$. Then, we have a congruence

$$
\operatorname{deg}\left(\Delta_{X}, \Delta_{X}\right)_{s} \equiv \operatorname{ord}\left(\operatorname{disc} H_{d R}^{n}\left(X_{K} / K\right)\right) \bmod 2 .
$$

We deduce Proposition 2.1 from Lemma 2.2 below. To state Lemma, we introduce some notation. For $X$ as in Proposition 2.1 , let $\omega_{X / O_{K}}=$ 
$\operatorname{det} \Omega_{X / O_{K}}^{1}$ be the relative canonical sheaf. It is an invertible $O_{X}$-module. There is a canonical map $c: \Omega_{X / O_{K}}^{n}=\Lambda^{n} \Omega_{X / O_{K}}^{1} \rightarrow \omega_{X / O_{K}}$. It is an isomorphism on the smooth generic fiber $X_{K}$.

A complex $C$ of $O_{X}$-modules is said to be perfect if the cohomology sheaves $\mathcal{H}^{q}(C)$ are coherent for all $q$ and if $\mathcal{H}^{q}(C)=0$ except for finitely many $q$. For a perfect complex $C$ of $O_{X}$-modules, its dual complex $D C=$ $R \mathcal{H} \operatorname{lom}_{O_{X}}\left(C, \omega_{X / O_{K}}\right)$ is also a perfect complex $C$ of $O_{X}$-modules. If a perfect complex $C$ is acyclic in positive degree i.e. $\mathcal{H}^{q}(C)=0$ for $q>0$, the dual $D C$ is acyclic in negative degree i.e. $\mathcal{H}^{q}(D C)=0$ for $q<0$. Hence a map $\mathcal{H}^{0}(C) \rightarrow \mathcal{H}^{\prime} m_{O_{X}}\left(\mathcal{H}^{0}(C), \omega_{X / O_{K}}\right)$ of coherent sheaves induces a map $C \rightarrow D C$ of perfect complexes. For a map $C \rightarrow C^{\prime}$ of perfect complexes, let Cone $\left[C \rightarrow C^{\prime}\right]$ denote the mapping cone. For a perfect complex $C$ of $O_{X^{-}}$ modules acyclic on the generic fiber $X_{K}$, the hypercohomology $H^{q}(X, C)$ are $O_{K}$-modules of finite length for all $q$ and are 0 except for finitely many $q$. We define its Euler number by

$$
\chi(X, C)=\sum_{q}(-1)^{q} \text { length }_{O_{K}} H^{q}(X, C) .
$$

Lemma 2.2. Let $X$ be as in Proposition 2.1 and put $n=2 m$. Then, there exists a perfect complex $C$ of $O_{X}$-modules satisfying the following properties (1) and (2).

(1) The complex $C$ is acyclic in positive degree, the 0-th cohomology sheaf is canonically isomorphic to $\Omega_{X / O_{K}}^{m}=\Lambda^{m} \Omega_{X / O_{K}}^{1}$ and the generic fiber $C \otimes_{O_{K}} K$ is acyclic except for degree 0 .

Let $b: C \rightarrow D C=R$ Hom $_{O_{X}}\left(C, \omega_{X / O_{K}}\right)$ be the map induced by the map

$$
\Omega_{X / O_{K}}^{m} \rightarrow \mathcal{H o m}_{O_{X}}\left(\Omega_{X / O_{K}}^{m}, \omega_{X / O_{K}}\right): x \mapsto(y \mapsto c(x \wedge y))
$$

and by the canonical isomorphism in (1).

(2) The mapping cone Cone $[C \rightarrow D C]$ is acyclic on the generic fiber $X_{K}$ and, for the Euler number, we have a congruence

$$
\operatorname{deg}\left(\Delta_{X}, \Delta_{X}\right)_{s} \equiv \chi(X, \text { Cone }[C \rightarrow D C]) \quad \bmod 2 .
$$

In section 4 , we define the derived exterior power complex $L \Lambda^{m} \Omega_{X / O_{K}}^{1}$ and prove that $C=L \Lambda^{m} \Omega_{X / O_{K}}^{1}$ satisfies the conditions (1) and (2) in Lemma 2.2 to complete the proof of congruence (0.1).

We deduce Proposition 2.1 from Lemma 2.2. By Lemma 2.2, it is sufficient to show

$$
\chi(X,[C \rightarrow D C]) \stackrel{(2.1)}{\equiv} \operatorname{ord} \operatorname{disc} H^{m}\left(X, \Omega_{X / K}^{m}\right) \stackrel{(2.2)}{\equiv} \operatorname{ord} \operatorname{disc} H_{d R}^{n}(X / K) .
$$

We prove the congruences (2.1) and (2.2). 
We show (2.1). By Grothendieck duality, the map $b: C \rightarrow D C$ induces a pairing

$$
H^{q}(X, C) \times H^{n-q}(X, C) \rightarrow H^{n}\left(X, \omega_{X / O_{K}}\right) \rightarrow O_{K}
$$

Tensoring $K$, we recover the cup-product

$$
H^{q}\left(X_{K}, \Omega_{X_{K} / K}^{m}\right) \times H^{n-q}\left(X, \Omega_{X_{K} / K}^{m}\right) \rightarrow H^{n}\left(X, \Omega_{X_{K} / K}^{n}\right) \rightarrow K .
$$

For an $O_{K}$-module $M$ of finite type, let $M^{\prime}$ denote the free quotient $M^{\prime}=$ $M / M_{\text {tors }}$ and $M^{*}$ denote the dual $M^{*}=\operatorname{Hom}_{O_{K}}\left(M, O_{K}\right)$. Let $H^{q}(b)^{\prime}$ denote the induced map

$$
H^{q}(b)^{\prime}: H^{q}(X, C)^{\prime} \longrightarrow H^{n-q}(X, C)^{\prime *}, x \mapsto(y \mapsto \operatorname{Tr}(x \cup y)) .
$$

For $q=m$, the free quotient $H^{m}(X, C)^{\prime}$ is a lattice of $H^{m}\left(X_{K}, \Omega_{X_{K} / K}^{m}\right)$ and hence we have a congruence

$$
\operatorname{ord}\left(\operatorname{disc} H^{m}\left(X_{K}, \Omega_{X_{K} / K}^{m}\right)\right) \equiv \text { length Coker } H^{m}(b)^{\prime} \quad \bmod 2 .
$$

On the other hand, by the exact sequence

$$
\begin{aligned}
0 \longrightarrow \operatorname{Hom}\left(H^{n-q+1}(X, C)_{\text {tors }}, K / O_{K}\right) & \longrightarrow H^{q}(X, D C) \\
& \longrightarrow H^{n-q}(X, C)^{\prime *} \longrightarrow 0,
\end{aligned}
$$

we have

$$
\begin{aligned}
& \chi(X,[C \rightarrow D C]) \\
& =\sum_{q}(-1)^{q}\left(\text { lengthCoker } H^{q}(b)^{\prime}\right. \\
& \left.+ \text { length } H^{n-q+1}(X, C)_{\text {tors }}-\text { length } H^{q}(X, C)_{\text {tors }}\right) \\
& =(-1)^{m} \text { length Coker } H^{m}(b)^{\prime}-2 \sum_{q}(-1)^{q} \text { length }\left(H^{q}(X, C)_{\text {tors }}\right) \\
& +\sum_{q<m}(-1)^{q}\left(\text { length Coker } H^{q}(b)^{\prime}+\text { length Coker } H^{n-q}(b)^{\prime}\right) .
\end{aligned}
$$

Since the pairings $H^{q}(X, C)^{\prime} \times H^{n-q}(X, C)^{\prime} \rightarrow O_{K}$ are $(-1)^{m-q_{-s y m m e t r i c}}$ to each other, the cokernel Coker $H^{q}(b)^{\prime}$ is isomorphic to Coker $H^{n-q}(b)^{\prime}$. Thus we have a congruence

$$
\chi(X,[C \rightarrow D C]) \equiv \text { length Coker } H^{m}(b)^{\prime} \bmod 2
$$

and hence the congruence (2.1) is proved.

We show (2.2). It follows from Lemma 2.3 below by taking the valuation.

Lemma 2.3. Let $K$ be an arbitrary field of characteristic $\neq 2$ and $X$ be a proper smooth scheme of even dimension $n=2 m$ over $K$. We put $b=\operatorname{dim} H_{d R}^{n}(X / K)$ and $h=\operatorname{dim} H^{m}\left(X, \Omega_{X / K}^{m}\right)$. Then, we have $\operatorname{disc} H_{d R}^{n}(X / K)=(-1)^{\frac{b-h}{2}} \operatorname{disc} H^{m}\left(X, \Omega_{X / K}^{m}\right)$ 
in $K^{\times} / K^{\times 2}$.

Proof of Lemma 2.3. By Proposition 4 and its Corollary in [11], we have $(-1)^{m b+\frac{b(b-1)}{2}} \operatorname{disc} H_{d R}^{n}(X / K)=(-1)^{m h+\frac{h(h-1)}{2}} \operatorname{disc} H^{m}\left(X, \Omega_{X / K}^{m}\right)$.

Since $b \equiv h \bmod 2$, the assertion follows.

\section{Parity of conductor and discriminant}

We prove the congruence $(0.2)$.

Proposition 3.1. Let $X$ be a projective smooth scheme of even dimension $n$ over a complete discrete valuation field $K$ with perfect residue field. If the characteristic of $K$ is not 2 , we have a congruence

$$
\operatorname{ord}\left(\operatorname{disc} H_{d R}^{n}(X / K)\right) \equiv \operatorname{Art}\left(\operatorname{det} H^{n}\left(X_{\bar{K}}, \mathbb{Q}_{\ell}\right)\right) \quad \bmod 2 .
$$

Proof. We recall the relation between the determinant of $\ell$-adic cohomology and the discriminant of de Rham cohomology. Let $X$ be a projective smooth scheme of even dimension $n=2 m$ over a field $K$ of characteristic $\neq 2, \ell$. Then the determinant of the orthogonal $\ell$-adic representation $H^{n}\left(X_{\bar{K}}, \mathbb{Q}_{\ell}\right)\left(\frac{n}{2}\right)$ defines a character $G_{K} \rightarrow\{ \pm 1\} \subset \mathbb{Q}_{\ell}^{\times}$of the absolute Galois group $G_{K}=\operatorname{Gal}(\bar{K} / K)$. We regard it as an element

$$
\operatorname{det} H^{n}\left(X_{\bar{K}}, \mathbb{Q}_{\ell}\right)\left(\frac{n}{2}\right) \in \operatorname{Hom}\left(G_{K},\{ \pm 1\}\right)=K^{\times} / K^{\times 2} .
$$

We identify $K^{\times} / K^{\times 2}$ with $\operatorname{Hom}\left(G_{K},\{ \pm 1\}\right)$ by the canonical map $a \mapsto$ $\chi_{a}=(\sigma \mapsto \sigma(\sqrt{a}) / \sqrt{a})$. Then we have

$$
\operatorname{Art} \chi_{a} \equiv \operatorname{ord} a \bmod 2 .
$$

In fact, the conductor Art $\chi_{a}$ is equal to the valuation ord $d_{K(\sqrt{a}) / K}$ of the discriminant of the quadratic extension $K(\sqrt{a})$. The class of the discriminant in $K^{\times} / K^{\times 2}$ is $4 a$ and the assertion follows.

By the congruence (3.1), Proposition 3.1 follows immediately from

Theorem 3.2. ([11] Theorem 2) Let $X$ be a projective smooth scheme of even dimension $n=2 m$ over a field $K$ of characteristic $\neq 2, \ell$. Then we have

$$
(-1)^{m \chi\left(X_{\bar{K}}\right)+b^{-}} \operatorname{disc} H_{d R}^{n}(X / K)=\operatorname{det} H^{n}\left(X_{\bar{K}}, \mathbb{Q}_{\ell}\right)\left(\frac{n}{2}\right)
$$

in $K^{\times} / K^{\times 2}$. Here $b^{-}=\sum_{i<n} \operatorname{dim} H_{d R}^{i}(X / K)$. 


\section{Derived exterior power complex}

In this section, we recall the definition of the derived exterior power complex $L \Lambda^{q} \Omega_{X / O_{K}}^{1}$ and show, Corollary 4.9 , that the complex $L \Lambda^{m} \Omega_{X / O_{K}}^{1}$ for $q=m=\frac{n}{2}$ satisfies the conditions (1) and (2) in Lemma 2.2 to complete the proof of Theorem 0.2 .

First, we recall the definition of the derived exterior power complex $L \Lambda^{q} K$ for a complex $K=[L \rightarrow E]$. The definition given in [10] is wrong and we give a correct definition, cf. Lemma 4.3. However the definition in [10] is correct if $L$ is an invertible sheaf, Example 4.2 below. Hence the results proved in [10] are true if we just replace the wrong definition there by the correct definition below for it is proved by reducing to the case where $L$ is invertible, cf. Proof of Proposition 4.8 below. After recalling the definition, we define another complex using symmetric tensors, which is canonically quasi-isomorphic to the derived exterior power complex. More systematic account will be given in [8].

Definition 4.1. Let $d: L \rightarrow E$ be a morphism of locally free $O_{X}$-modules of finite rank on a scheme $X$ and let $K=[L \stackrel{d}{\rightarrow} E]$ be the chain complex where $E$ is put on degree 0 . For an integer $q \in \mathbb{Z}$, we define the derived exterior power complex $L \Lambda^{q} K=\left(\left(L \Lambda^{q} K\right)_{n}, d_{n}\right)$.

For an integer $n$, we define the $n$-th component $\left(L \Lambda^{q} K\right)_{n} \subset \Lambda^{q}\left(E \oplus L^{\oplus n}\right)$ to be the direct summand

$$
\begin{aligned}
\left(L \Lambda^{q} K\right)_{n}= & \bigoplus{ }_{\sum_{i=0}^{n} q_{i}=q}{ }^{\prime} \Lambda^{q_{0}} E \otimes \Lambda^{q_{1}} L \otimes \cdots \otimes \Lambda^{q_{n}} L \\
\subset \Lambda^{q}\left(E \oplus L^{\oplus n}\right)= & \bigoplus_{\sum_{i=0}^{n} q_{i}=q} \Lambda^{q_{0}} E \otimes \Lambda^{q_{1}} L \otimes \cdots \otimes \Lambda^{q_{n}} L
\end{aligned}
$$

where the integers $q_{0}, \ldots, q_{n}$ in $\bigoplus^{\prime}$ run those satisfying the condition $q_{i}>0$ for $1 \leq i \leq n$. We have $\left(L \Lambda^{q} K\right)_{n}=0$ unless $\max (0,(q-\operatorname{rank} E) / \operatorname{rank} L) \leq$ $n \leq q$.

We define the boundary map $d_{n}:\left(L \Lambda^{q} K\right)_{n} \rightarrow\left(L \Lambda^{q} K\right)_{n-1}$ to be the restriction $d_{n}=\left.\left(\Lambda^{q} \tilde{d}_{n}\right)\right|_{\left(L \Lambda^{q} K\right)_{n}}$ of the $q$-th exterior power of the map

$$
\tilde{d}_{n}: E \oplus L^{\oplus n} \rightarrow E \oplus L^{\oplus n-1}
$$

defined by $\left(x_{0}, x_{1}, x_{2}, \ldots, x_{n}\right) \mapsto\left(x_{0}+\tilde{d}\left(x_{1}\right), x_{2}-x_{1}, \ldots, x_{n}-x_{1}\right)$. Since $\tilde{d}_{n-1} \circ \tilde{d}_{n}\left(x_{0}, x_{1}, x_{2}, \ldots, x_{n}\right)=\tilde{d}_{n-1}\left(x_{0}, x_{2}, \ldots, x_{n}\right)$, they satisfy $d_{n-1} \circ d_{n}=$ 0 .

For $q<0$, the complex $L \Lambda^{q} K$ is 0.

Example 4.2. If $L$ is an invertible module, the complex $L \Lambda^{q} K$ is

$$
\left[L^{\otimes q} \rightarrow E \otimes L^{\otimes q-1} \rightarrow \cdots \rightarrow \Lambda^{q-n} E \otimes L^{\otimes n} \rightarrow \cdots \rightarrow \Lambda^{q} E\right]
$$

with the boundary $\operatorname{map} d_{n}\left(x_{0} \otimes x_{1}^{\otimes n}\right)=\left(x_{0} \wedge d x_{1}\right) \otimes x_{1}^{\otimes n-1}$. 
The 0-th homology sheaf $L^{0} \Lambda^{q} K=\mathcal{H}_{0}\left(L \Lambda^{q} K\right)$ is canonically isomorphic to the $q$-th exterior power $\Lambda^{q} \mathcal{H}_{0} K$ of the cokernel $\mathcal{H}_{0} K=\operatorname{Coker}(d: L \rightarrow$ $E)$. In fact, $d_{1}:\left(L \Lambda^{q} K\right)_{1} \rightarrow\left(L \Lambda^{q} K\right)_{0}$ is

$$
\oplus_{q_{0}} \operatorname{id} \wedge \Lambda^{q-q_{0}} d: \bigoplus_{0 \leq q_{0}<q} \Lambda^{q_{0}} E \otimes \Lambda^{q-q_{0}} L \rightarrow \Lambda^{q} E .
$$

We verify that Definition 4.1 is a special case of the general definition of the derived functor of the exterior power by Dold-Puppe [4] (cf. [7]), in the case where the complex is of amplitude $[-1,0]$. Let the notation be as in 1.3 [7] with the following exception. To avoid confusion, we use the letter $T$ to denote the Dold-Puppe transform which is denoted by $K$ in the reference.

Lemma 4.3. Let $K=[L \stackrel{d}{\rightarrow} E]$ be a complex as in Definition 4.1 and $q \geq 0$ be an integer. Then, the normal complex $N \Lambda^{q} T(K)$ of the derived $q$-th exterior power $\Lambda^{q} T(K)$ of the Dold-Puppe transform $T(K)$ of the complex $K$ is canonically isomorphic to the complex $L \Lambda^{q} K$ defined above.

Proof. We keep the notation in $1.3[7]$ with the exception stated above. Following the definition given there, the Dold-Puppe transform $T(K)$ is computed as follows. The $n$-th component $T(K)_{n}$ is $E \oplus L^{\oplus n}$. Its 0-th component $E$ in $E \oplus L^{\oplus n}$ corresponds to the map $\epsilon_{0}:[0, n] \rightarrow[0]$ and, for $1 \leq i \leq n$, the $i$-th $L$ corresponds to the surjection $\epsilon_{i}:[0, n] \rightarrow[0,1]$ defined by $\epsilon_{i}(j)=j$ for $j<i$ and $\epsilon_{i}(j)=j+1$ for $j \geq i$. Each component of the boundary map $d_{i}: E \oplus L^{\oplus n} \rightarrow E \oplus L^{\oplus n-1}$ for $0 \leq i \leq n$ is described as follows.

On $E$, the identity to $E$ for all $i$.

On $j$-th $L$ for $1 \leq j \leq n$,

the identity map to $j$-th $L$ if $j \leq i$ except for $j=i=n$,

the identity map to $(j-1)$-st $L$ if $j>i$ except for $j-1=i=0$, the 0 -map for $j=i=n$ and

$d: L \rightarrow E$ for $j=1, i=0$.

If $1 \leq i<n$, the map $d_{i}$ looks like

$$
\begin{array}{cccccccc}
E & \oplus & L & \oplus \cdots \oplus & \stackrel{i}{\rightarrow} L & \oplus \stackrel{i+1}{\rightarrow} L & \oplus \cdots \oplus \stackrel{n-1}{\rightarrow} L & \oplus \stackrel{n}{\rightarrow} L \\
\operatorname{id} \downarrow & & \text { id } \downarrow & & \text { id } \downarrow & \swarrow \text { id } & \cdots \swarrow / \text { id } & \swarrow \text { id } \\
E & \oplus & L & \oplus \cdots \oplus & \underset{i}{\rightarrow} L & \oplus \underset{i+1}{\rightarrow} L & \oplus \cdots \oplus \underset{n-1}{\rightarrow} L . &
\end{array}
$$

The description above is a consequence of the following fact. For $0 \leq i \leq n$, let $\delta_{i}:[0, n-1] \rightarrow[0, n]$ be the map defined by $\delta_{i}(j)=j$ for $j<i$ and $\delta_{i}(j)=j+1$ for $j \geq i$. Then we have $\delta_{i} \circ \epsilon_{j}=\epsilon_{j}$ for $j \leq i$ except for $j=i=n, \delta_{i} \circ \epsilon_{j}=\epsilon_{j-1}$ for $j>i$ and $\delta_{n} \circ \epsilon_{n}=\epsilon_{0}$. 
To describe the $q$-th exterior power, we introduce an isomorphism

$$
\begin{aligned}
\alpha_{n}: E \oplus L^{\oplus n} \rightarrow T(K)_{n} & =E \oplus L^{\oplus n}, \\
& \left(x_{0}, x_{1}, x_{2}, \ldots, x_{n}\right) \mapsto\left(x_{0}, x_{1}, x_{2}-x_{1}, x_{3}-x_{2}, \ldots, x_{n}-x_{n-1}\right) .
\end{aligned}
$$

We identify $T(K)_{n}$ with $E \oplus L^{\oplus n}$ by $\alpha_{n}$. Then the composite map $d_{i}^{\prime}=$ $\alpha_{n-1}^{-1} \circ d_{i} \circ \alpha_{n}: E \oplus L^{\oplus n} \rightarrow E \oplus L^{\oplus n-1}$ is simply the map droping the $i$-th component $L$ for $1 \leq i \leq n$. Since the inverse $\alpha_{n}^{-1}$ is given by $\left(x_{0}, x_{1}, x_{2}, \ldots, x_{n}\right) \mapsto\left(x_{0}, x_{1}, x_{2}+x_{1}, \ldots, \sum_{i=1}^{n} x_{i}\right)$, the map $d_{0}^{\prime}$ is the same as the map $\tilde{d}:\left(x_{0}, x_{1}, x_{2}, \ldots, x_{n}\right) \mapsto\left(x_{0}+d\left(x_{1}\right), x_{2}-x_{1}, x_{3}-x_{1}, \ldots, x_{n}-x_{1}\right)$ in Definition 4.1.

We compute the normal complex $N \Lambda^{q} T(K)$. By its definition, the $n$ th component $N \Lambda^{q} T(K)_{n}$ is $\bigcap_{i=1}^{n} \operatorname{Ker}\left(d_{i}: T(K)_{n} \rightarrow T(K)_{n-1}\right)$. By the isomorphism $\alpha_{n}$ above, it is identified with

$$
\bigcap_{i=1}^{n} \operatorname{Ker}\left(d_{i}^{\prime}: \Lambda^{q}\left(E \oplus L^{\oplus n}\right) \rightarrow \Lambda^{q}\left(E \oplus L^{\oplus n-1}\right)\right)=\left(L \Lambda^{q} K\right)_{n} \subset \Lambda^{q}\left(E \oplus L^{\oplus n}\right) .
$$

By $d_{0}^{\prime}=\tilde{d}$, the boundary maps are the same as that in Definition 4.1 and Lemma 4.3 is proved.

Corollary 4.4. Let $K=[L \rightarrow E]$ and $K^{\prime}=\left[L^{\prime} \rightarrow E^{\prime}\right]$ be complexes as above.

(1). For two morphisms $f, g: K=[L \rightarrow E] \rightarrow K^{\prime}=\left[L^{\prime} \rightarrow E^{\prime}\right]$ homotope to each other, there exists a homotopy between the $L \Lambda^{q}(f), L \Lambda^{q}(g)$ : $L \Lambda^{q} K \rightarrow L \Lambda^{q} K^{\prime}$.

(2) If $f: K=[L \rightarrow E] \rightarrow K^{\prime}=\left[L^{\prime} \rightarrow E^{\prime}\right]$ is a quasi-isomorphism, the induced map $L \Lambda^{q}(f): L \Lambda^{q} K \rightarrow L \Lambda^{q} K^{\prime}$ is also a quasi-isomorphism.

Proof. (1) is a consequence of the general fact that the functors $N$ and $T$ preserve homotopy. For (2), since the question is local on $X$, we may assume $f$ has an inverse $g: K^{\prime} \rightarrow K$ upto homotopy. Then, (1) implies (2).

For an integer $q \in \mathbb{Z}$ and a complex $K=[L \rightarrow E]$ as above, we define another complex $L^{\prime} \Lambda^{q} K$ using symmetric tensors. We give a canonical quasi-isomorphism $L^{\prime} \Lambda^{q} K \rightarrow L \Lambda^{q} K$ in Lemma 4.6 below.

Definition 4.5. 1. For a quasi-coherent $O_{X}$-module $M$ and an integer $n$, the $n$-th symmetric tensor module $T S^{n} M$ is the fixed part

$$
T S^{n} M=\bigcap_{\sigma \in \mathcal{S}_{n}} \operatorname{Ker}\left(\sigma-1: M^{\otimes n} \rightarrow M^{\otimes n}\right)
$$

by the natural action of the symmetric group $\mathcal{S}_{n}$.

2. Let $K=[E \rightarrow L]$ be a complex of locally free $O_{X}$-modules of finite rank. For an integer $q, n \geq 0$, we put $\left(L^{\prime} \Lambda^{q} K\right)_{n}=\Lambda^{q-n} E \otimes T S^{n} L$. We 
define a $\operatorname{map}_{n}: \Lambda^{q-n} E \otimes T S^{n} L \rightarrow \Lambda^{q-n+1} E \otimes T S^{n-1} L$ to be the restriction of

$$
\begin{aligned}
& \Lambda^{q-n} E \otimes L^{\otimes n} \rightarrow \Lambda^{q-n+1} E \otimes L^{\otimes n-1}: \\
& x_{0} \otimes x_{1} \otimes x_{2} \otimes \cdots \otimes x_{n} \mapsto\left(x_{0} \wedge d x_{1}\right) \otimes x_{2} \otimes \cdots \otimes x_{n} .
\end{aligned}
$$

The $\mathcal{S}_{n}$-invariance implies the $\mathcal{S}_{n-1}$-invariance and that $d_{n-1} \circ d_{n}=0$. The complex $L^{\prime} \Lambda^{q} K$ is defined to be

$$
\begin{aligned}
& {\left[T S^{q} L \stackrel{d_{q}}{\longrightarrow} E \otimes T S^{q-1} L \longrightarrow \quad \cdots \quad \longrightarrow \Lambda^{q-n} E \otimes T S^{n} L\right.} \\
& \left.\stackrel{d_{n}}{\longrightarrow} \quad \ldots \quad \longrightarrow \Lambda^{q-1} E \otimes L \stackrel{d_{1}}{\longrightarrow} \Lambda^{q} E\right] .
\end{aligned}
$$

The last term $\Lambda^{q} E$ is put on degree 0 . For $q<0$, we put $L^{\prime} \Lambda^{q} K=0$.

Lemma 4.6. Let $K=[L \stackrel{d}{\rightarrow} \stackrel{d}{\rightarrow} E]$ be a complex as in Definition 4.1 and $q \in \mathbb{Z}$ be an integer. Then, the inclusion $\left(L^{\prime} \Lambda^{q} K\right)_{n}=\Lambda^{q-n} E \otimes T S^{n} L \subset \Lambda^{q-n} E \otimes L^{\otimes n} \subset\left(L \Lambda^{q} K\right)_{n}\left(\subset \Lambda^{q}\left(E \oplus L^{\oplus n}\right)\right)$ induces a quasi-isomorphism

$$
L^{\prime} \Lambda^{q} K \rightarrow L \Lambda^{q} K
$$

If $L$ is an invertible $O_{X}$-module, the canonical map $L^{\prime} \Lambda^{q} K \rightarrow L \Lambda^{q} K$ is the identity (cf. Example 4.2).

Proof. First, let us note that $T S^{r} L$ is the kernel of the map $L^{\otimes r} \rightarrow$ $\bigoplus_{i=2}^{r} L^{\otimes i-2} \otimes \Lambda^{2} L \otimes L^{\otimes r-i}$ defined by

$x_{1} \otimes x_{2} \otimes \cdots \otimes x_{n} \mapsto \sum_{i=2}^{r}(-1)^{i+1} x_{2} \otimes \cdots \otimes x_{i-1} \otimes\left(x_{1} \wedge x_{i}\right) \otimes x_{i+1} \otimes \cdots \otimes x_{n}$.

We show that the inclusions are compatible with the boundary and hence defines a canonical map $L^{\prime} \Lambda^{q} K \rightarrow L \Lambda^{q} K$. By the definition of the boundary map, we have an equality

$$
\begin{aligned}
& d\left(x_{0} \otimes x_{1} \otimes x_{2} \otimes \cdots \otimes x_{n}\right)-\left(x_{0} \wedge d x_{1}\right) \otimes x_{2} \otimes \cdots \otimes x_{n} \\
= & \sum(-1)^{i+1} x_{0} \otimes x_{2} \otimes \cdots \otimes x_{i-1} \otimes\left(x_{1} \wedge x_{i}\right) \otimes x_{i+1} \otimes \cdots \otimes x_{n}
\end{aligned}
$$

for $x_{0} \otimes x_{1} \otimes x_{2} \otimes \cdots \otimes x_{n} \in \Lambda^{q} E \otimes L^{\otimes n}$. Hence the assertion follows from the remark above.

We prove that the canonical map $L^{\prime} \Lambda^{q} K \rightarrow L \Lambda^{q} K$ is a quasi-isomorphism. We define a decreasing filtration $F$ on $L \Lambda^{q} K$. Let

$$
F^{r}\left(L \Lambda^{q} K\right)_{n}=\bigoplus_{q_{0} \geq r}^{\prime} \Lambda^{q_{0}} E \otimes \Lambda^{q_{1}} L \otimes \cdots \otimes \Lambda^{q_{n}} L \subset\left(L \Lambda^{q} K\right)_{n}
$$

where $\bigoplus_{q_{0} \geq r}{ }^{\prime}$ means that we add the condition $q_{0} \geq r$ to the condition in $\bigoplus^{\prime}$ in Definition 4.1. We have $F^{0}\left(L \Lambda^{q} K\right)_{n}=\left(L \Lambda^{q} K\right)_{n}, F^{q-n}\left(L \Lambda^{q} K\right)_{n}=$ 
$\Lambda^{q-n} E \otimes L^{\otimes n}$ and $F^{q-n+1}\left(L \Lambda^{q} K\right)_{n}=0$. It is easily seen to define a filtration on the complex $L \Lambda^{q} K$. The graded piece $G r_{F}^{q-r}\left(L \Lambda^{q} K\right)$ is the same as $\Lambda^{q-r} E \otimes L \Lambda^{r}([L \rightarrow 0])$. Therefore to prove Lemma, it is enough to show that the inclusion $T S^{r} L \rightarrow L^{\otimes r}$ induces a quasi-isomorphism $T S^{r} L[r] \rightarrow L \Lambda^{r}([L \rightarrow 0])$. By Proposition 4.3.2.1 [7] Chap.1, there is a quasi-isomorphism $T S^{r} L[r] \rightarrow L \Lambda^{r}([L \rightarrow 0])$. Thus it is also reduced to the fact in the beginning of the proof.

The author does not know if the functor $L^{\prime} \Lambda^{q}$ preserves homotopy.

We extend the complex $L \Lambda^{q} K$ to a complex denoted by $\bar{L} \Lambda^{q} K$ with a canonical map $\bar{L} \Lambda^{q} K \rightarrow L \Lambda^{q} K$.

Definition 4.7. Let $K=[L \stackrel{d}{\rightarrow} E]$ be a complex as above.

The rank $r=r_{K}$ of $K$ is the difference $r_{E}(=\operatorname{rank} E)-r_{L}(=\operatorname{rank} L)$.

The determinant $\operatorname{det} K$ of $K$ is defined to be the invertible $O_{X}$-module $\mathcal{H o m}\left(\Lambda^{r_{L}} L, \Lambda^{r_{E}} E\right)$.

For an integer $q \in \mathbb{Z}$, the extended derived exterior power complex $\bar{L} \Lambda^{q} K$ is defined to be the mapping fiber

$$
\bar{L} \Lambda^{q} K=\operatorname{Fiber}\left[L \Lambda^{q} K \rightarrow \mathcal{H o m}\left(L \Lambda^{r-q} K, \operatorname{det} K\right)\right] .
$$

of the map $L \Lambda^{q} K \rightarrow \mathcal{H}$ om $\left(L \Lambda^{r-q} K, \operatorname{det} K\right)$ induced by the canonical map

$$
\Lambda^{q} E \rightarrow \mathcal{H o m}\left(\Lambda^{r-q} E, \operatorname{det} K\right): e \mapsto\left(e^{\prime} \mapsto\left(\ell \mapsto e \wedge e^{\prime} \wedge d \ell\right)\right) .
$$

The map $\Lambda^{q} E \rightarrow \mathcal{H o m}\left(\Lambda^{r-q} E, \operatorname{det} K\right)$ is the 0-map unless $0 \leq q \leq r_{K}$. It is the same as the compositum

$$
\Lambda^{q} E \rightarrow \Lambda^{q} \mathcal{H}_{0}(K) \rightarrow \mathcal{H o m}\left(\Lambda^{r-q} \mathcal{H}_{0}(K), \operatorname{det} K\right) \rightarrow \mathcal{H o m}\left(\Lambda^{r-q} E, \operatorname{det} K\right) .
$$

By Lemma 4.2, the complex $\bar{L} \Lambda^{q} K$ is canonically quasi-isomorphic to the complex

$$
\begin{gathered}
{\left[T S^{q} L \rightarrow \cdots \rightarrow \Lambda^{q} E \rightarrow \mathcal{H o m}\left(\Lambda^{r-q} E, \operatorname{det} K\right) \rightarrow\right.} \\
\left.\quad \cdots \rightarrow \mathcal{H o m}\left(T S^{r-q} L, \operatorname{det} K\right)\right] \\
\simeq\left[T S^{q} L \rightarrow \cdots \rightarrow \Lambda^{q} E \rightarrow \mathcal{H o m}\left(\Lambda^{r_{L}} L, \Lambda^{r_{L}+q} E\right) \rightarrow\right. \\
\left.\cdots \rightarrow \mathcal{H o m}\left(\Lambda^{r_{L}} L \otimes T S^{r-q} L, \Lambda^{r_{E}} E\right)\right]
\end{gathered}
$$

where $\Lambda^{q} E$ is put on degree 0 . There is a canonical map $\bar{L} \Lambda^{q} K \rightarrow L \Lambda^{q} K$. For $q=r=r_{K}$, the complex $\bar{L} \Lambda^{r} K$ defined here is the same as $\bar{L} \Lambda^{r} K$ defined in (1.5) in [10].

The extended derived exterior power complex $\bar{L} \Lambda^{q} K$ satisfies similar properties, Proposition 4.8 below, as $\bar{L} \Lambda^{r_{K}} K$ proved in [10]. To state it, we introduce some notations. For a complex $K=[L \stackrel{d}{\rightarrow} E]$ as above, let $Z=$ $Z\left(\Lambda^{r_{L}} d\right) \subset X$ be the closed subscheme defined as the zero locus of $\Lambda^{r_{L}} d$. Namely, locally on $X$, the closed subscheme $Z$ is defined by the determinant of $r_{L} \times r_{L}$-minors of a matrix representing the map $d: L \rightarrow E$. On the 
complement $X-Z$, the cokernel $\mathcal{H}_{0}(K)=\operatorname{Coker}(d: E \rightarrow L)$ is locally free of rank $r=r_{K}$ and the natural map $K \rightarrow \mathcal{H}_{0}(K)$ is a quasi-isomorphism. Bloch defines in [1] a localized Chern class $c_{r+1} X(K) \in C H^{r+1}(Z \rightarrow X)$. An element of $C H^{t}(Z \rightarrow X)$ is a functorial families of homomorphisms $C H_{s}(Y) \rightarrow C H_{s-t}\left(Y \times_{X} Z\right)$ for all $Y \rightarrow X$. For a perfect complex $M$ of $O_{X}$-modules acyclic outside a closed subscheme $Z \subset X$, the localized Chern class $c_{s} X(M) \in C H^{s}(Z \rightarrow X)$ is defined in [6] Section 18.1. Slightly generalizing the results in [10], we have the following Proposition 4.8.

Proposition 4.8. Let $q \in \mathbb{Z}$ and $K=[L \stackrel{d}{\rightarrow} E]$ be a complex of locally free $O_{X}$-modules of finite rank as above. We assume the following condition is satisfied.

(L). Locally on $X$, the complex $K$ is quasi-isomorphic to a complex $K^{\prime}=$ $\left[L^{\prime} \stackrel{d^{\prime}}{\rightarrow} E^{\prime}\right]$ where $E^{\prime}$ is locally free of finite rank and $L^{\prime}$ is invertible. Then,

(1). The cohomology sheaves $\mathcal{H}_{j}\left(\bar{L} \Lambda^{q} K\right)$ are $O_{Z}$-modules for all $j \in \mathbb{Z}$. The sheaves $\mathcal{H}_{q-r_{K}}\left(\bar{L} \Lambda^{q} K\right)$ and $L^{1} i^{*} K=\mathcal{T}$ or ${ }_{1}^{O_{X}}\left(K, O_{Z}\right)$ are invertible $O_{Z}$ modules. There is a canonical isomorphism of $\mathrm{O}_{Z}$-modules

$$
L^{1} i^{*} K \otimes_{O_{Z}} \mathcal{H}_{j}\left(\bar{L} \Lambda^{q} K\right) \rightarrow \mathcal{H}_{j+1}\left(\bar{L} \Lambda^{q+1} K\right) .
$$

(2). For the localized Chern classes, we have

$$
c_{m X}^{Z}\left(\bar{L} \Lambda^{q} K\right)= \begin{cases}0 & \text { if } m \leq r_{K} \\ (-1)^{q-1} r_{K} ! c_{m_{X}}^{Z}(K) & \text { if } m=r_{K}+1\end{cases}
$$

in $C H^{m}(Z \rightarrow X)$. The left hand side is that defined in [6] and the right hand side is that defined in [1].

Assume $X$ is projective and locally of complete intersection over a discrete valuation ring $O_{K}$ and $\operatorname{dim} X=r_{K}+1$. Assume further that the support of $Z$ is a subset of the closed fiber $X_{F}$. Then,

(3). The complex $\bar{L} \Lambda^{q} K$ is acyclic on the generic fiber $X_{K}$. For the Euler characteristic $\chi\left(X, \bar{L} \Lambda^{q} K\right)=\sum_{j}(-1)^{j}$ length ${ }_{O_{K}} \mathcal{H}^{j}\left(X, \bar{L} \Lambda^{q} K\right)$, we have a formula

$$
\chi\left(X, \bar{L} \Lambda^{q} K\right)=(-1)^{q-\left(r_{K}+1\right)} \operatorname{deg} c_{r_{K}+1}^{Z}(K) \cap[X] .
$$

Proof. Proof is similar to those in [10]. Since the proofs in [10] is done by reducing to the case where the $O_{X}$-module $L$ is invertible for which the definition given there is correct, the error in the definition of the complex in [10] has no influence on the proof as we will see below.

(1). It is proved similarly as [10] Proposition 1.7. Since the assertion is local on $X$, we may assume the sheaf $L$ is invertible by the assumption (L) and by Corollary 4.4 (2) . Then, as in Lemma (1.6) [10], it follows 
immediately from the definition that we have

$$
L \otimes \bar{L} \Lambda^{q} K[1]=\bar{L} \Lambda^{q+1} K .
$$

From this, we obtain the isomorphism in (1). Further the rest of the assertions are reduced to the case $q=n$, which is [10] Proposition 1.7.

(2). It is proved similarly as [10] Proposition 2.1. By the same argument as there, it is enough to show that $\bar{L} \Lambda^{q} \tilde{K}$ is acyclic except for degree $r+$ $1-q$ and the homology sheaf $\mathcal{H}_{q-(r+1)} \bar{L} \Lambda^{q} \tilde{K}$ is an invertible $O_{\tilde{D}}$-module in the notation there. Since the assertion is proved for $q=r+1$ in [10] Lemma 2.2 , it is enough to show that $\bar{L} \Lambda^{q} \tilde{K}$ is locally quasi-isomorphic to $\bar{L} \Lambda^{r+1} \tilde{K}[q-(r+1)]$. By the condition (L), by Corollary $4.4(2)$ and by the isomorphism (4.1) above, the assertion is proved for all $q$.

(3). The proof is the same as [10] Proposition 2.3, with Proposition 2.1 in it replaced by the assertion (2) here.

We define the derived exterior power complex for a quasi-coherent $O_{X^{-}}$ module $M$ satisfying the following condition.

(G). There exists a resolution $0 \rightarrow L \rightarrow E \rightarrow M \rightarrow 0$ by locally free $O_{X}$-modules $E$ and $L$ of finite rank.

We define the derived exterior power complex $L \Lambda^{q} M$ to be $L \Lambda^{q}[L \rightarrow E]$ by taking a resolution as in $(\mathrm{G})$. It is well-defined upto homotopy, since such a resolution is unique upto homotopy. In particular, the cohomology sheaves $L^{s} \Lambda^{q} M$ are well-defined upto unique canonical isomorphism. The determinant $\operatorname{det} M$, the rank $r_{M}$ and the extended derived exterior power complex $\bar{L} \Lambda^{q} M=\left[L \Lambda^{q} M \rightarrow R \mathcal{H o m}\left(L \Lambda^{r_{M}-q} M, \operatorname{det} M\right)\right]$ are similarly defined as above.

We may apply Proposition 4.8 to the complex $\bar{L} \Lambda^{q} M$, if $M$ further satisfies the condition

(L'). Locally on $X$, there exists a resolution $0 \rightarrow L^{\prime} \rightarrow E^{\prime} \rightarrow M \rightarrow 0$ by a locally free $O_{X}$-module $E$ of finite rank and an invertible $O_{X}$-module $L$. In this case, the closed subscheme $Z \subset X$ is defined by the annihilater ideal of $\Lambda^{r_{M}+1} M$ by Proposition 4.8 (1).

We apply Proposition 4.8 to $M=\Omega_{X / O_{K}}^{1}$. Let $X$ be a regular flat projective scheme over a discrete valuation ring $O_{K}$ with perfect field $F$ such that the generic fiber $X_{K}$ is smooth of dimension $r$. We verify that the $O_{X}$-module $M=\Omega_{X / O_{K}}^{1}$ satisfies the conditions $(\mathrm{G})$ and $(\mathrm{L})$ above. Since we assume $X$ is a subscheme of $P=\mathbb{P}_{O_{K}}^{N}$ for some integer $N$, we have an exact sequence

$$
\left.0 \longrightarrow N_{X / P} \longrightarrow \Omega_{P / S}^{1}\right|_{X} \longrightarrow \Omega_{X / S}^{1} \longrightarrow 0
$$

and the condition $(\mathrm{G})$ is satisfied. Locally on $X$, there is a regular immersion $X \rightarrow P^{\prime}$ of codimension 1 to a smooth scheme $P^{\prime}$ over $O_{K}$. A similar exact sequence for this immersion implies the condition (L'). Thus 
the derived exterior power complex $L \Lambda^{q} \Omega_{X / O_{K}}^{1}$ is defined and we may apply Proposition 4.8 to it.

To show Lemma 2.2, we identify the extended derived exterior power complex $\bar{L} \Lambda^{q} \Omega_{X / O_{K}}^{1}$. Recall that $\omega_{X / O_{K}}=\operatorname{det} \Omega_{X / O_{K}}^{1}$ is the relative canonical sheaf. The wedge product

$$
\Omega_{X / O_{K}}^{q} \otimes \Omega_{X / O_{K}}^{r-q} \rightarrow \Omega_{X / O_{K}}^{r} \rightarrow \omega_{X / O_{K}}
$$

induces a map $\Omega_{X / O_{K}}^{q} \rightarrow R \mathcal{H}$ om $_{O_{X}}\left(\Omega_{X / O_{K}}^{r-q}, \omega_{X / O_{K}}\right)$ and hence a map

$$
b: L \Lambda^{q} \Omega_{X / O_{K}}^{1} \rightarrow D_{X}\left(L \Lambda^{r-q} \Omega_{X / O_{K}}^{1}\right)=R \mathcal{H} \operatorname{Hom}_{O_{X}}\left(L \Lambda^{r-q} \Omega_{X / O_{K}}^{1}, \omega_{X / O_{K}}\right)
$$

as in Lemma 2.1. The map $b$ is the same as the map in the definition of $\bar{L} \Lambda^{q} \Omega_{X / O_{K}}^{1}$. Hence the complex $\bar{L} \Lambda^{q} \Omega_{X / O_{K}}^{1}$ is identified with the mapping fiber of the map $b$ induced by the wedge product:

$$
\bar{L} \Lambda^{q} \Omega_{X / O_{K}}^{1}=\text { Fiber }\left[L \Lambda^{q} \Omega_{X / O_{K}}^{1} \rightarrow D_{X}\left(L \Lambda^{r-q} \Omega_{X / O_{K}}^{1}\right)\right] .
$$

Corollary 4.9. Let $X$ be a regular flat projective scheme over a discrete valuation ring $O_{K}$ with perfect field $F$ such that the generic fiber $X_{K}$ is smooth of dimension $r$ and $q \in \mathbb{Z}$ be an integer. Then, the mapping fiber Fiber $\left[L \Lambda^{q} \Omega_{X / O_{K}}^{1} \rightarrow D_{X}\left(L \Lambda^{r-q} \Omega_{X / O_{K}}^{1}\right)\right]$ is acyclic on the generic fiber and we have a formula for the Euler number:

$$
(-1)^{q} \operatorname{deg}\left(\Delta_{X}, \Delta_{X}\right)=\chi\left(X, \text { Fiber }\left[L \Lambda^{q} \Omega_{X / O_{K}}^{1} \rightarrow D_{X}\left(L \Lambda^{r-q} \Omega_{X / O_{K}}^{1}\right)\right]\right) .
$$

Proof. We identify the mapping fiber

$$
\text { Fiber }\left[L \Lambda^{q} \Omega_{X / O_{K}}^{1} \rightarrow D_{X}\left(L \Lambda^{r-q} \Omega_{X / O_{K}}^{1}\right)\right]
$$

with the extended derived power complex $\bar{L} \Lambda^{q} \Omega_{X / O_{K}}^{1}$. Since we assume the generic fiber is smooth, the support of $Z$ is a subset of the closed fiber $X_{F}$. Hence the complex $\bar{L} \Lambda^{q} \Omega_{X / O_{K}}^{1}$ is acyclic on the generic fiber $X_{K}$ and the Euler number is defined. Now it is enough to apply Proposition 4.8 (3) to the $O_{X}$-module $M=\Omega_{X / O_{K}}^{1}$.

Proof of Lemma 2.2. We verify that $C=L \Lambda^{m} \Omega_{X / O_{K}}^{1}$ satisfies the conditions (1) and (2) in Lemma 2.2. The condition (1) follows immediately from the definition of the exterior derived power complex. Since the Euler number of the mapping cone is the minus of that of the mapping fiber, the condition (2) is a consequence of Corollary 4.9.

Thus the proof of Theorem 0.2 is completed.

\section{References}

[1] S. BlOCH, Cycles on arithmetic schemes and Euler characteristics of curves. Proc. Symp. Pure Math. AMS 46 Part 2 (1987), 421-450.

[2] - De Rham cohomology and conductors of curves. Duke Math. J. 54 (1987), 295-308.

[3] P. Deligne (after A. Grothendieck), Résumé des premiers exposés de A.Grothendieck. in SGA7I, Lecture notes in Math. 288, Springer, 1-23. 
[4] A. Dold, D. PuPPE, Homologie nicht additiver Funktoren. Ann. Inst. Fourier 11 (1961), 201-312.

[5] K. FujIWARA, A proof of the absolute purity conjecture (after Gabber). Algebraic geometry 2000, Azumino (Hotaka), 153-183, Adv. Stud. Pure Math. 36, Math. Soc. Japan, Tokyo, 2002

[6] W. Fulton, Intersection theory. Springer.

[7] L. Illusie, Complexe cotangent et déformations I. Lecture notes in Math. 239 Springer.

[8] K. Kato, T. SAITO, Conductor formula of Bloch. To appear in Publ. Math. IHES.

[9] T. OCHIAI, $\ell$-independence of the trace of monodromy. Math. Ann. 315 (1999), 321-340.

[10] T. SAITo, Self-intersection 0-cycles and coherent sheaves on arithmetic schemes. Duke Math. J. 57 (1988), 555-578.

[11] — Jacobi sum Hecke characters, de Rham discriminant, and the determinant of $\ell$-adic cohomologies. J. of Alg. Geom. 3 (1994), 411-434.

[12] J.-P. SERRE, Conducteurs d'Artin des caracteres réels. Inventiones Math. 14 (1971), 173183.

Takeshi SAITo

Department of Mathematical Sciences,

University of Tokyo

Tokyo 153-8914 Japan

E-mail : t-saito@ms.u-tokyo.ac.jp 